\title{
A New Method of Surgical Enterocele Correction Using Mesh Implants
}

DOI: $10.17691 / \mathrm{stm} 2017.9 .3 .10$

Received December 29, 2016

A.A. Ischenko, MD, PhD, Head of Oncogynecology Unit, University Clinical Hospital No.4;

L.S. Aleksandrov, MD, DSc, Professor, Department of Obstetrics and Gynecology No.1.

I.D. Hokhlova, MD, PhD, Associate Professor, Department of Obstetrics and Gynecology No. $1^{1-}$

Y.N. Tarasenko, MD, PhD, Tutor, Department of Obstetrics and Gynecology No. 1';

E.P. Hudoley, PhD Student, Department of Obstetrics and Gynecology No.1';

Y.Y. Sulina, PhD Student, Department of Obstetrics and Gynecology No.11;

T.V. Gavrilova, Obstetrician-Gynecologist, Gynecology Unit²;

A.I. Ischenko, MD, DSc, Professor, Head of the Department of Obstetrics and Gynecology No. $1^{1}$; Director ${ }^{2}$

II.M. Sechenov First Moscow State Medical University, 8/2 Trubetskaya St., Moscow, 119991, Russian Federation;

${ }^{2}$ V.F. Snegirev Clinic of Obstetrics and Gynecology, $2 / 1$ Yelanskogo St., Moscow, 119435, Russian Federation

The aim of the investigation was to develop and evaluate a new method of enterocele correction by laparovaginal access.

Materials and Methods. 23 women treated for the recurrent pelvic organ prolapse participated in the study. Group 1 included 13 patients after total hysterectomy by the vaginal and laparotomic access with concurrent correction of cystocele using mesh implants, group 2 consisted of 10 patients after organ-saving operations on the small pelvic organs in combination with ventrosuspension of the uterus by aponeurotic flaps and colpoperineoplasty and levatorplasty.

Results. Findings of the present study confirm the concept of polyetiology of pelvic organ prolapse and the increasing risk of enterocele formation following the operation, leading to impairment of normal anatomical interposition of the small pelvic organs and damage to the fascial and ligamentous apparatus. The developed method of a single-step, two-level laparovaginal correction of enterocele using mesh implants with their multipoint fixation is highly efficient, its application helps to improve operative treatment results and reduce the disease recurrence rate.

Key words: enterocele correction; pelvic organ prolapse; meshes.

In the structure of gynecological diseases, pelvic prolapses make $28.0-38.9 \%$ [1]. The incidence of prolapses grows with the increase of life duration reaching tenfold progression in postmenopausal relative to the reproductive period promoted by hypoestrogenia causing atrophy in estrogen-dependent pelvic organs and tissues [2].

Of a special clinical interest is a separate group of pelvic prolapses, enterocele, which is characterized as a hernia of pelvic parietal peritoneum containing the loops of the small intestine and/or omentum. Enterocele generates as a result of thinning or rupture of rectovaginal and/or pubocervical fascia allowing the peritoneum to move closely to the vaginal mucous membrane. Most commonly, the overstretched peritoneum of the rectouterine pouch participates in enterocele formation leading to sinking-down of the posterior fornix of the vagina and sometimes to the prolapse of the latter together with the hernial sac beyond the pudendal fissure [3].

Factors predisposing to enterocele formation are deep rectouterine space, operative interventions on the pelvic organs accompanied by the damage of pubocervical and/or rectovaginal fascia as well as impairment of normal anatomical interposition of the pelvic organs after hysterectomy, uterus ventrofixation, other methods of prolapse correction [4].

Despite numerous operative techniques by vaginal and abdominal access, the recurrence rate of pelvic organ prolapse remains high reaching 33.3-40.0\% [5].

Functional inefficiency of quite a number of operative interventions, which is frequently connected with incomplete surgical reconstruction of normal interposition of the pelvic organs, insufficient correction of fascial defects, inadequate volume of the operation, is frequently reported $[5,6]$.

The aim of the investigation was to develop and evaluate a new method of enterocele correction by laparovaginal access.

Materials and Methods. The work was conducted from 2013 to 2016 in V.F. Snegirev Clinic of Obstetrics and Gynecology, a clinical base of the Department of Obstetrics and Gynecology No.1 of I.M. Sechenov First Moscow State Medical University of the Ministry of Health

For contacts: Anton A. Ischenko, e-mail: ra2001_2001@mail.ru 
of the Russian Federation, in the Medical Rehabilitation Center of the Ministry of Health of the Russian Federation, in PriorClinic of Reproductive Health, Moscow.

A complex examination and surgical treatment were performed to 23 women with recurrent prolapse of pelvic organs 6-15 years after the first operative intervention.

Patients were divided into two groups based on the volume of the previous operations. Group 1 included 13 patients after total hysterectomy performed by the vaginal and laparotomic access with concurrent correction of cystocele with mesh implants, group 2 consisted of 10 patients after organ-saving operations on the small pelvic organs in combination with ventrosuspension of the uterus by aponeurotic flaps and colpoperineoplasty and levatorplasty.

All patients were in the period of postmenopause, duration of which varied from 2 to 21 years. The mean age was 59.6 years, of them $47.8 \%$ were at the age of 54-59 years, the rest $(52.2 \%)$ were 60-67 years old.

The study complies with the Declaration of Helsinki (the Declaration was passed in June 1964, Helsinki, Finland, and revised in October 2000, Edinburgh, Scotland) and was performed following approval by the Ethics Committee of I.M. Sechenov First Moscow State Medical University. Written informed consent was obtained from every patient.

The examined groups were formed using continuous thematic sample method. Isolated forms of enterocele after the previous operative interventions accompanied by correction of various forms of pelvic prolapse served as criteria of inclusion into the study. Patients with a combination of enterocele with other kinds of pelvic organ prolapses, with severe extragenital pathology, and absolute contraindications to laparoscopy were excluded from the investigation.

Women of both groups were examined in compliance with the standards of rendering medical aid. They included complaint analysis, history taking, physical examination, gynecological and rectal investigations, instrumental methods (transvaginal, transperineal ultrasound, colposcopy), and other methods of investigation, which were administered depending on the individual clinical symptoms and necessary preoperative preparation.

Analyzing patients' histories, special attention was paid to the character of the complaints and the time of their onset, heredity, extragenital pathology, specifics of menstrual and reproductive functions, the character of gynecological pathology and operative interventions in the past, history of the present disease.

During clinical investigations, the vagina and cervix of the uterus were inspected with the help of specula, and then gynecological examination was carried out, which is the basis of diagnosing pelvic organ prolapse. Rectal examination helped to determine presence or absence of the posterior vaginal wall descent, and differentiate enterocele from rectocele. To determine the prolapse degree, the international pelvic organ prolapse quantification system was used. Echographic examination of pelvic organs was performed using Toshiba aplio apparatus (Toshiba, Japan), provided with multifrequency transvaginal and convex detectors.

A detailed analysis of complaints showed that they were similar in both groups. First of all, the patients noted discomfort and the sense of a foreign body in the vagina $(100 \%)$, nagging pain in the lower parts of the abdomen and lower back (91.3\%), uneasiness and unpleasant sensation during intercourse (82.6\%) and, in this connection, inability of adequate sexual life. Constipation occurred in $21.7 \%$ of patients. Four women $(17.4 \%)$ noted a fallout of a foreign body, which was persistently called a uterus, beyond the vagina limits in a standing position, during straining efforts and physical exertion, and in walking.

Three females from the group 1 experienced initial vaginal discomfort 5 years after the operation; five females experienced it post 7 years; four females post 8 years and one case of initial vaginal discomfort was registered 12 years after the operation. Identical complaints appeared from four females from the group 26 years after the operation; from three females - post 7 years; and from other three females 8 years after the operation.

But the majority of patients sought medical aid only 3-6 years later trying to avoid secondary operations and hoping for a conservative treatment.

Family history study revealed presence of pelvic prolapse in mothers in each fourth case, in maternal grandmothers in each sixth patient, which may speak in favor of the hereditary character of the disease [7].

Among the extragenital pathology, $47.8 \%$ of patients had cardiovascular diseases, $17.4 \%$ varicose disease, $21.7 \%$ chronic bronchitis, $26 \%$ chronic gastritis and chronic cholecystitis, $8.7 \%$ hernias, $13 \%$ nephroptosis, $17.4 \%$ joint hypermobility and dislocations, $30.4 \%$ arthrosis, $73.9 \%$ osteochondrosis of various parts of the spine, assuming systemic disorders of the connective tissue structure $[8,9]$.

Menstrual function and menopause age in patients of both groups were comparable and without any specific features. Thus, the age of menarche was on average $13.0 \pm 1.4$ years, menopause $51.4 \pm 3.6$ years.

In $82.6 \%$ of patients, there were $1-2$, in $17.4 \% 3$ term deliveries through the natural maternal passages. Labor complicated by perineal trauma was in $60.8 \%$ of women, which is also a risk factor for the development of pelvic organ prolapse $[10,11]$.

Among gynecological diseases hysteromyoma was noted in the history of 17 women, benign serous cystadenoma in 4, and ovary teratoma in 2 patients. Additionally, cystocele was diagnosed in the past in patients of group 1, and in patients of group 2 II-III stage colpoptosis was found.

At the age of 42-54 years, all patients underwent operative treatment: total hysterectomy and correction of cystocele by a mesh implant (group 1); 
myomectomy in 4, uni-, bilateral adnexectomy in 6 patients by laparotomic access in combination with ventrosuspension of the uterus and colpoperineoplasty and levatorplasty (group 2).

During gynecological and rectal examination, there were no signs of pelvic floor incompetence as evidenced by a good perineal condition and closed pudendal fissure in $82.6 \%$ of patients. Strained efforts opened the fissure and the apical part of the hernial sac originating from the upper third of the vagina became available for visualization. In $17.4 \%$ of patients the hernial sac prolapsed partially beyond the vagina. When the latter was reduced inwardly, the fissure closed. The data obtained speak of a pathogenetic character of enterocele formation, i.e. of the main role of overstretching and/ or ruptures of the rectovaginal fascia [10]. Descent or bulging of the middle or lower third of the posterior vaginal wall as well as rectocele were not registered.

Transvaginal and transperineal echography showed the picture characteristic for the unaltered pelvic floor. Cervix of the uterus in patients of group 2 was visualized above or at the level of symphysis pubis, the height of the tendinous center of the perineum was less than $10 \mathrm{~mm}$, muscular diastasis in the area of the tendinous center was absent. Deformation of the contours and displacement of the bladder and the rectum were not noted. Valsalva test did not show urethral hypermobility.

At the same time, bulging coming from the area located higher than the level of the serrated line of the anal canal, in which there were intestinal loops and/or omentum, was visualized in the vagina.

Hysteromyoma was found in 4 patients of group 2: from 2 to 4 nodes $1-2 \mathrm{~cm}$ in diameter with interstitially subserous localization.

Recurrence of pelvic prolapse was an indication to the operative treatment - an isolated form of III stage (in 19) and IV stage (in 4) enterocele.

Results. Surgery is the most effective method of treating pelvic prolapse. Despite the availability of multiple correcting techniques, there is no universal operation solving all the problems of prolapse, the recurrence rate for this disease is high, therefore, surgeons of related specialties (gynecologists, urologists, proctologists) are constantly developing new methods, the application of which, sometimes in combination with each other, allows them to obtain not only good but also stable results $[1,5]$.

Relying on the knowledge and skills of the surgeons of previous generation, investigations of contemporary scientists, our own practical experience, and the latest medical technologies, we developed an original method of treating enterocele - single-step, two-level correction using mesh implants.

There are three well-known basic surgical techniques of abdominal enterocele correction: Moshkovich, Galban operations, and placation of sacrouterine ligaments, which assume obliteration of recto-uterine pouch. The main technique by vaginal access includes identification of the defect in the area of the upper and lateral parts of the recto-uterine fascia, through which organs of the abdominal cavity, usually omentum and/or small bowel loops, prolapse into the vagina, with the following dissection and excision of the hernial sac, suturing the hernial orifice, and elimination of the fascial defect [1].

We used laparovaginal access performing singlestep, two-level correction of enterocele using mesh implants with preliminary adhesiolysis, since adhesive process extension in the small pelvis was detected to be grade I-II in 15, and grade III in 8 patients.

Patients of group 1 underwent adhesiolysis, transvaginal extraperitoneal sacrocolpopexy, sacrospinous colpopexy.

Adhesiolysis, transvaginal extraperitoneal sacrocervicocolpopexy, sacrospinal colpopexy and supravaginal amputation of uterus were performed to the patients of group 2. To improve the access to the operative field, perineotomy with the following colpoperineoplasty and levatorplasty was done in 4 patients.

The operative intervention was carried out in several stages.

1. Operation was started with the revision of pelvic organs, adhesiolysis, examination of hernial sac presented by the distended peritoneal rectouterine pouch, and evacuation of its content (loops of the small bowel, omentum) by laparoscopic access.

2. Concurrently, the vaginal wall above the enterocele was fixed by clamps, and after hydropreparation vaginal mucous membrane was dissected in the longitudinal direction up to the peritoneum avoiding damage to it. Then the hernial sac was dissected to its orifice, facilitated by laparoscopic transillumination. From the front, we oriented to the level of the cervix of the uterus (group 2) or the lower edge of the symphysis pubis (group 1), posteriorly orientation was to the level of the coccyx, from the right and left to the level of ischial spines. During dissection the hernial sac was carefully separated from the anterior wall of the rectum. Canals to the sacrospinal ligaments were formed bilaterally by sharp and blunt techniques.

3. Extraperitoneal tunnel from the dome (group 1) or posterior vault (group 2) of the vagina to the sacropromontory under the parietal peritoneum of the posterior pelvic wall to the right from the midline was formed by transvaginal access under the endoscopic control with the help of long soft forceps. The peritoneum was opened over the promontory and emptied from the adjacent cellular tissue by laparoscopic access. Then a mesh implant $(3 \times 15 \mathrm{~cm}$ proline mesh) was brought transvaginally through the extraperitoneal tunnel to the sacropromontory, the proximal end of the implant was fixed by 1-2 nonabsorbable endosutures to the longitudinal sacral ligament in the area of the promontory. The distal end of the mesh was anchored to the superior part of the posterior wall of the vagina (group 1) and to the posterior wall of the uterine cervix and to the posterior vaginal vault (group 2) using vaginal access. 
4. The peritoneum of the empty hernial sac was pulled up by endoscopic forceps higher to the pelvic cavity, thereafter the sac orifice was ligated by endoscopic looped sutures and additional endosutures fixing the empty hernial sac to the posterior wall of the uterine cervix (group 2) and one of the sacrouterine ligaments (group 1 and 2). Then placation of the sacrouterine ligaments was performed by $2-4$ endosutures to reduce the depth of the Douglas pouch.

5. Concurrently, two rows of purse-string sutures were placed transvaginaly on the area of the hernial orifice, and thereafter sacrospinal colpopexy was performed using proline mesh implant $9.0 \pm 1.0 \mathrm{~cm}$ long and $4.0 \pm 1.0 \mathrm{~cm}$ wide with $8 \times 2 \mathrm{~cm}$ sleeves, and Elevate posterior system (AMS, USA). With the help of guiding devices the sleeves of the Elevate posterior system were fixed to the sacrospinal ligaments from both sides $2 \mathrm{~cm}$ from the ischiadic spines in the medial direction. The sleeves were inserted in the special openings of the mesh implant turned reversely, the angles of which were immersed into the wound with a pusher, and then additional fixators were placed. Besides, the mesh implant was ligated by separate sutures to the posterior wall of the uterine cervix (group 2), posterior vaginal wall (group 1 and 2), rectovaginal fascia, and a distal end of the implant fixed previously to the sacropromontory. Multipoint fixation of the mesh implants helps prevent their curling and shrinkage leading to better results of the operation and a long-term therapeutic effect. The excessive mucous membrane of the posterior wall of the vaginal upper third was then ablated, and separate vicryl sutures were placed on the wound.

6. Supravaginal amputation of the uterus was performed to the patients of group 2 by laparoscopic access using a conventional technique.

Operations in groups 1 and 2 lasted on average for $137.8 \pm 25.0 \mathrm{~min}$ and varied from 75 to $240 \mathrm{~min}$ (group 1) and from 110 to 190 min (group 2). Intraoperative blood loss was $169.2 \pm 32.9 \mathrm{ml}$ in patients of group 1 , and $145.0 \pm 22.7 \mathrm{ml}$ in group 2 .

There were no complications in the early postoperative period.

The analysis of the data obtained during the outpatient follow-up in the late postoperative period (3-36 months) showed that subjectively patients were satisfied with the results.

During gynecological examination, transvaginal, and transperineal echography it was noted that the vaginal dome, the first and posterior vaults were high in the patients of group 2. Straining efforts did not cause significant displacements of the pelvic organs.

Conclusion. Findings of the present study confirm the concept of polyetiology of pelvic organ prolapse and the increasing risk of enterocele formation following the operation, leading to impairment of normal anatomical interposition of the small pelvic organs and damage to the fascial and ligamentous apparatus. The developed method of a single-step, two-level laparovaginal correction of enterocele using mesh implants with their multipoint fixation is highly efficient, its application helps to improve operative treatment results and reduce the disease recurrence rate.

Study Funding and Conflicts of Interest. The work was not supported by any sources, and there are no conflicts of interest related to the present study.

\section{References}

1. Ahmad M., Sileri P., Franceschilli L., Mercer-Jones M. The role of biologics in pelvic floor surgery. Colorectal Dis 2012; 14(Suppl 3): 19-23, https://doi.org/10.1111/codi.12045.

2. Ruiz-Zapata A.M., Kerkhof M.H., Zandieh-Doulabi B., Brölmann H.A., Smit T.H., Helder M.N. Fibroblasts from women with pelvic organ prolapse show differential mechanoresponses depending on surface substrates. Int Urogynecol J 2013; 24(9): 1567-1575, https://doi.org/10.1007/s00192-013-2069-z.

3. Morris V.C., Murray M.P., Delancey J.O., AshtonMiller J.A. A comparison of the effect of age on levator ani and obturator internus muscle cross-sectional areas and volumes in nulliparous women. Neurourol Urodyn 2012; 31(4): 481486, https://doi.org/10.1002/nau.21208.

4. Jelovsek J.E., Chagin K., Brubaker L., Rogers R.G., Richter H.E., Arya L., Barber M.D., Shepherd J.P., Nolen T.L., Norton P., Sung V., Menefee S., Siddiqui N., Meikle S.F., Kattan M.W.; Pelvic Floor Disorders Network. A model for predicting the risk of de novo stress urinary incontinence in women undergoing pelvic organ prolapse surgery. Obstet Gynecol 2014; 123(2 Pt 1): 279-287, https://doi.org/10.1097/ aog.0000000000000094.

5. Strizhakov A.N., Kosachenko A.G., Davydov A.I. Genital hernias. The current state of the problem. Voprosy ginekologii, akušerstva $i$ perinatologii 2016; 15(1): 58-64, https://doi. org/10.20953/1726-1678-2016-1-58-64.

6. Larson K.A., Luo J., Guire K.E., Chen L., AshtonMiller J.A., DeLancey J.O. 3D analysis of cystoceles using magnetic resonance imaging assessing midline, paravaginal, and apical defects. Int Urogynecol J 2012; 23(3): 285-293, https://doi.org/10.1007/s00192-011-1586-x.

7. Ward R.M., Velez Edwards D.R., Edwards T., Giri A., Jerome R.N., Wu J.M. Genetic epidemiology of pelvic organ prolapse: a systematic review. Am J Obstet Gynecol 2014; 211(4): 326-335, https://doi.org/10.1016/j.ajog.2014.04.006.

8. Wang S., Zhang Z., Lü D., Xu Q. Effects of mechanical stretching on the morphology and cytoskeleton of vaginal fibroblasts from women with pelvic organ prolapse. Int J Mol Sci 2015; 16(5): 9406-9419, https://doi.org/10.3390/ijms16059406.

9. Egorov V., van Raalte H., Lucente V. Quantifying vaginal tissue elasticity under normal and prolapse conditions by tactile imaging. Int Urogynecol J 2012; 23(4): 459-466, https://doi.org/10.1007/s00192-011-1592-z.

10. Ulrich D., Edwards S.L., Su K., White J.F., Ramshaw J.A., Jenkin G., Deprest J., Rosamilia A., Werkmeister J.A., Gargett C.E. Influence of reproductive status on tissue composition and biomechanical properties of ovine vagina. PLoS One 2014; 9(4): e93172, https://doi. org/10.1055/s-0034-1374740.

11. Ren S., Xie B., Wang J., Rong Q. Three-dimensional modeling of the pelvic floor support systems of subjects with and without pelvic organ prolapse. Biomed Res Int 2015; 2015: 84598, https://doi.org/10.1155/2015/845985. 